\title{
Prevalência e fatores de risco associados à infecção da Esquistossomose mansoni e das enteroparasitoses em área endêmica, Sergipe, Brasil
}

\author{
Prevalence and risk factors associated with the infection of Schistosomiasis mansoni and \\ enteroparasitosis in an endemic area, Sergipe, Brazil \\ Predominio y factores de riesgo asociados a la infección por Schistosomiasis mansoni y \\ enteroparasitosis en una zona endémica, Sergipe, Brasil
}

Recebido: 01/04/2021 | Revisado: 10/04/2021 | Aceito: 21/04/2021 | Publicado: 06/05/2021

\author{
Alexrangel Henrique Cruz Santos \\ ORCID: https://orcid.org/0000-0002-3667-5275 \\ Federal University of Sergipe, Brazil \\ E-mail:alex_rangel2020@hotmail.com \\ Luciene Barbosa \\ ORCID: https://orcid.org/0000-0003-2026-994X \\ Federal University of Sergipe, Brazil \\ E-mail: lucienebarb@bol.com.br \\ Thayane Santos Siqueira \\ ORCID: https://orcid.org/0000-0001-8370-0544 \\ Federal University of Sergipe, Brazil \\ E-mail: thayane_siqueira@live.com \\ Mariana Rosário de Souza \\ ORCID: https://orcid.org/0000-0002-3139-8483 \\ Federal University of Sergipe, Brazil \\ E-mail:enf.mariama.rosario@gmail.com \\ Ariel Oliveira Celestino \\ ORCID: https://orcid.org/0000-0003-3907-187X \\ Federal University of Sergipe, Brazil \\ E-mail: biomédica.ariel@gmail.com \\ Amanda Francielle Santos \\ ORCID: https://orcid.org/0000-0001-7694-4604 \\ Federal University of Sergipe, Brazil \\ E-mail: francyelly_amanda@hotmail.com \\ Karina Conceição Gomes Machado De Araújo \\ ORCID: https://orcid.org/0000-0003-4433-5227 \\ Federal University of Sergipe, Brazil \\ E-mail:kkkaraujo2006@yahoo.com.br
}

\begin{abstract}
Resumo
Analisar a positividade e os fatores de risco associados à infecção da esquistossomose mansoni e das enteroparasitoses em área endêmica, Sergipe, Brasil. Trata-se de um estudo transversal. O estudo envolveu 160 moradores do povoado Siebra, situado na zona rural do município de Maruim, Sergipe. As positividades para Schistosoma mansoni e/ou enteroparasitos foram identificadas por meio do método Kato-Katz e do método de Ritchie, respectivamente, e correlacionados com as condições socioambientais da região. Em relação a positividade para esquistossomose foi de 49,37\%; a infecção prevaleceu no sexo masculino (52,70\%); em idosos (77,77\%). Os maiores riscos de se infectar com Schistosoma mansoni foi em indivíduos do sexo masculino, em idade (até 9 a $>40$ anos), que residem em área rural, recebem ajuda governamental, que tem constante contato com águas naturais e que recebem informações sobre as parasitoses. Quanto às amostras analisadas para as enteroparasitoses, os protozoários Endolimax nana e Giardia lamblia $(97,52 \%$ e $78,75 \%)$ foram os mais prevalentes; o Endolimax nana esteve mais prevalente em todas as faixas; a infecção prevaleceu no sexo feminino (42,52\%) para Trichuris trichiura, 9,19\% para Enterobius vermiculares, $67,81 \%$ para Entamoeba histolytica, 85,05\% para Giardia lamblia, 88,50\% para Entamoeba coli e 86,20\% para Blastocytis hominis). O estudo revelou que Maruim é endêmico para a esquistossomose e para as enteroparasitoses e que as condições socioambientais e sanitárias contribuem com as altas taxas de positividade. Os resultados encontrados mostram a necessidade do fortalecimento de programas de saúde com o objetivo de reduzir a transmissão e sensibilizar a população em relação a essas doenças.
\end{abstract}

Palavras-chave: Doenças tropicais negligenciadas; Saúde pública; Epidemiologia. 


\begin{abstract}
To analyze the positivity and risk factors associated with infection of schistosomesis mansoni and enteroparasitosis in an endemic area, Sergipe, Brazil. This is a cross-sectional study. The study involved 160 residents of the village of Siebra, located in the rural area of the municipality of Maruim, Sergipe. The positivities for Schistosoma mansoni and/or enteroparasites were identified using the Kato-Katz method and ritchie's method, respectively, and correlated with the socioenvironmental conditions of the region. Regarding the positivity for schistosomes was $49.37 \%$; infection prevailed in males $(52.70 \%)$; in the elderly $(77.77 \%)$. The greatest risks of becoming infected with Schistosoma mansoni were in male individuals, of age (up to 9 to $>40$ years), who live in rural areas, receive government help, who have constant contact with natural waters and who receive information about parasitosis. As for the samples analyzed for enteroparasitosis, the protozoa Endolimax nana and Giardia lamblia $(97.52 \%$ and $78.75 \%)$ were the most prevalent; Endolimax nana was more prevalent in all ranges; infection prevailed in females $(42.52 \%)$ for Trichuris trichiura, $9.19 \%$ for Enterobius vermiculars, $67.81 \%$ for Entamoeba histolytica, $85.05 \%$ for Giardia lamblia, $88.50 \%$ for Entamoeba coli and $86.20 \%$ for Blastocytis hominis). The study revealed that Maruim is endemic for schistostosis and enteroparasitasis and that socio-environmental and sanitary conditions contribute to high positivity rates. The results show the need to strengthen health programs with the objective of reducing transmission and sensitizing the population in relation to these diseases.
\end{abstract}

Keywords: Neglected tropical diseases; Public health; Epidemiology.

\title{
Resumen
}

Analizar la positividad y los factores de riesgo asociados con la infección de schistosomesis mansoni y enteroparasitosis en una zona endémica, Sergipe, Brasil. Este es un estudio transversal. En el estudio participaron 160 vecinos de la localidad de Siebra, situada en la zona rural del municipio de Maruim, Sergipe. Las positividades de Schistosoma mansoni y/o enteroparasitos fueron identificadas utilizando el método Kato-Katz y el método de Ritchie, respectivamente, y correlacionadas con las condiciones socioambientales de la región. En cuanto a la positividad de los cistosomas fue del 49,37\%; la infección prevaleció en los hombres (52,70\%); en los ancianos (77,77\%). Los mayores riesgos de infectarse con Schistosoma mansoni fueron en individuos varones, de edad (de hasta 9 a $>40$ años), que viven en zonas rurales, reciben ayuda del gobierno, que tienen contacto constante con aguas naturales y que reciben información sobre parasitosis. En cuanto a las muestras analizadas para enteroparasitosis, los protozoa Endolimax nana y Giardia lamblia $(97,52 \%$ y 78,75\%) fueron los más frecuentes; Endolimax nana era más frecuente en todos los rangos; infección prevaleció en las mujeres (42,52\%) para Trichuris trichiura, 9,19\% para Enterobius vermiculares, 67,81\% para Entamoeba histolytica, 85,05\% para Giardia lamblia, 88,50\% para Entamoeba coli y 86,20\% para Blastocytis hominis). El estudio reveló que Maruim es endémica de la eschistostosis y la enteroparasitasis y que las condiciones socioambientales y sanitarias contribuyen a altas tasas de positividad. Los resultados muestran la necesidad de fortalecer los programas de salud con el objetivo de reducir la transmisión y sensibilizar a la población en relación con estas enfermedades.

Palabras clave: Enfermedades tropicales desatendidas; Salud pública; Epidemiología.

\section{Introdução}

As parasitoses constituem um grave problema de saúde pública por possuírem uma significativa participação na morbidade e mortalidade mundial. Sua presença é um marcador típico de pobreza onde o acesso ao saneamento básico e água potável são limitados e, concomitantemente, os padrões de pobreza relativamente altos (Dunn, et al., 2016).

A esquistossomose mansoni causada pelo agente etiológico Schistosoma mansoni é uma doença de ocorrência tropical, típica da América do Sul, África e Antilhas (Brasil, 2017) popularmente conhecida como barriga d'água tendo como hospedeiro intermediário três espécies de caramujos: Biomphalaria tenagophila, B. glabrata e B straminea (Brasil, 2014).

De acordo com a Organização Mundial de Saúde a esquistossomose afeta quase 240 milhões de pessoas em todo o mundo e mais de 700 milhões de pessoas vivem em áreas endêmicas (Gomes, et al., 2016). No Brasil, as áreas endêmicas e focais atingem 19 unidades federativas. Acredita-se que cerca de 1,5 milhões de pessoas estejam infectadas pelo $S$. mansoni sendo os estados das regiões Nordeste e Sudeste os mais afetados (Brasil, 2012).

Em Sergipe, segundo o Programa de Controle da Esquistossomose (PCE), a esquistossomose mansoni prevalece em 51 dos 75 municípios (Machado, 2017) sendo as espécies B. glabrata e B. straminea responsáveis pela manutenção da doença (Fernandez, 2009). No período de 2005 e 2014, foram realizados 896.373 exames de fezes nos municípios endêmicos de Sergipe a maior prevalência da doença ocorreu no ano de 2008 (10,4\%) e a menor (6,4\%) em 2014 (Santos, et al., 2016).

As parasitoses intestinais ou enteroparasitoses possuem alta distribuição geográfica e apresenta altas taxas de contaminação. No Brasil representam um grave problema de saúde pública, particularmente, nos países subdesenvolvidos onde 
se apresentam bastante disseminadas e com alta prevalência, decorrente das más condições de vida das camadas populacionais mais carentes (Pereira, et al., 2017). A falta de orientação e conscientização higiênica induz a alta incidência dessa contaminação (Fochesatto Filho \& Barros, 2013).

Diversos programas governamentais em diversos países têm sido implementados para o controle dessas parasitoses. Nos países subdesenvolvidos a baixa eficácia de tais iniciativas está vinculada ao baixo investimento para medidas de adoção de saneamento básico e a falta da participação da comunidade (Dunn, et al., 2016). No Brasil, mais da metade das crianças em idade pré-escolar e escolar encontra-se parasitada ((Reuter, 2015). A falta de saneamento básico que envolve serviços de abastecimento público de água, de coleta de resíduos, de rede geral de esgotamento sanitário, ocasiona má disposição dos dejetos e inadequado destino do lixo, portanto, um ambiente poluído, o que propícia a incidência elevada de doenças parasitárias e o desenvolvimento de um agente infeccioso no homem ou animal (Fochesatto Filho \& Barros, 2013). Com base neste pressuposto, o presente estudo visava analisar a positividade e os fatores de risco associados a infecção da esquistossomose mansoni e das enteroparasitoses em área endêmica, Sergipe, Brasil

\section{Metodologia}

\subsection{Desenho do Estudo}

O presente estudo consistiu em uma pesquisa de cunho epidemiológico e transversal com abordagem descritiva. O corte transversal estuda a doença e exposição a fatores de risco de uma população em um dado momento do tempo (Pereira, et al., 2018). Na presente pesquisa, o corte se aplicou para identificação de moradores portadores de S. mansoni e enteroparasitos. Esse tipo de estudo evidenciou dados de prevalência e de múltiplos fatores de risco para essas infecções, não havendo necessidade de um período de seguimento. Sergipe é um dos nove estados localizados na região nordeste do Brasil. O município de Maruim se estende por $95,554 \mathrm{~km}^{2}$, com uma população de 16. 343 pessoas no ano de 2010 , sendo 12.437 residente na área urbana, 3.906 na área rural e uma população estimada de 17.271 habitantes para o ano de 2020. (IBGE, 2019). O estudo foi realizado no Povoado Siebra localizado na zona rural do município de Maruim (Figura 1). 
Figura 1. A) Mapa do Brasil com ênfase no estado de Sergipe. B) Mapa do estado de Sergipe com destaque ao município de Maruim. C) Mapa do munícipio de Maruim.

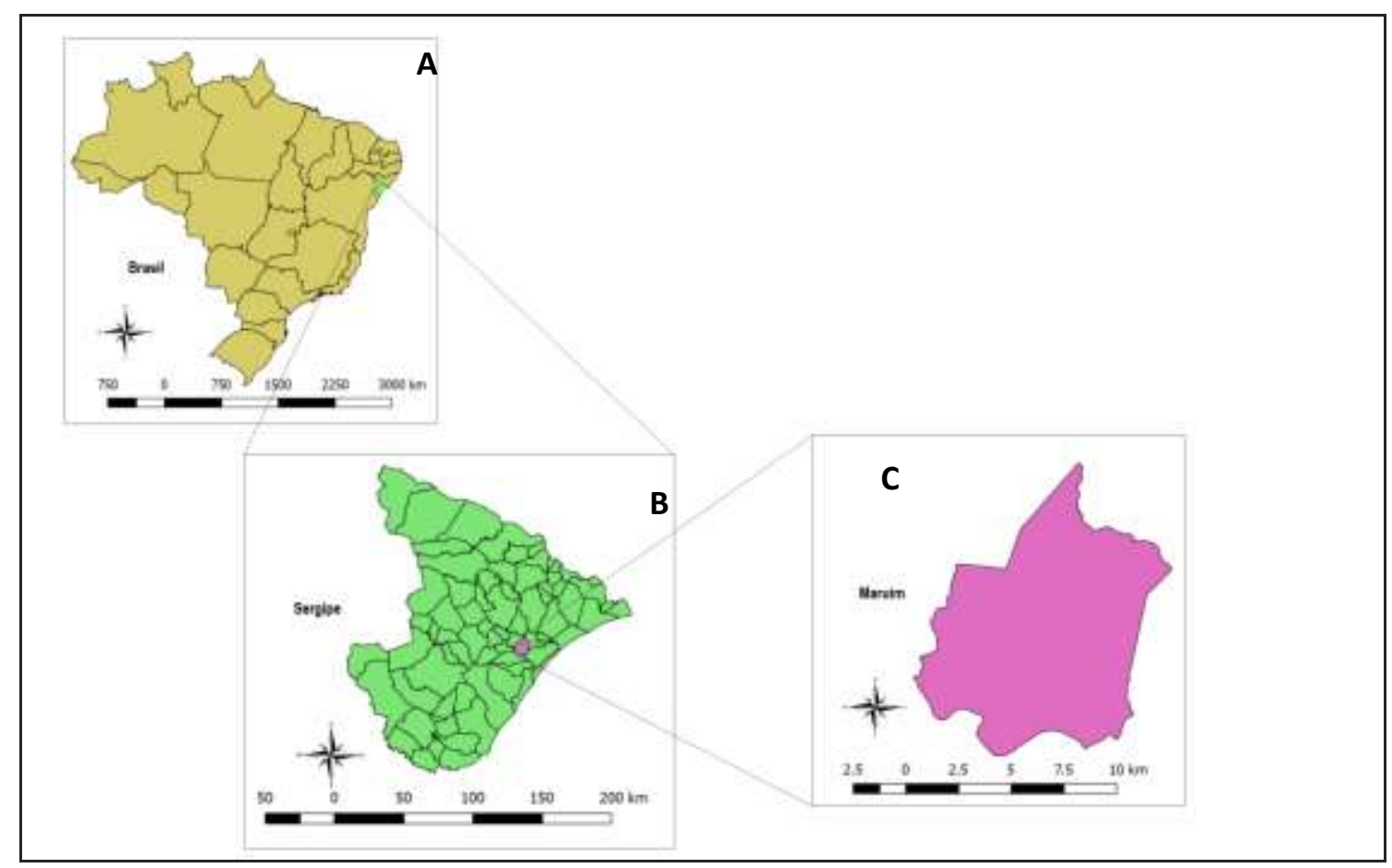

*Fonte: Autores.

Foi realizado um inquérito parasitológico através dos métodos de Kato-Katz e pelo método de Ritchie. Participaram do estudo 160 moradores. Para o planejamento, foi utilizado um cadastro prévio dos residentes do povoado e das respectivas residências, explicado o objetivo da pesquisa, entregue e recolhido a assinaturas dos termos de autorização (Termo de Consentimento e Assentimento do Menor). Um dia antes do início das coletas o primeiro coletor foi entregue aos participantes.

No primeiro dia, foi recolhido o primeiro frasco coletor com fezes, na medida que os moradores entregavam as amostras, recebiam novos coletores vazios para coletar nos dias seguintes computando um total de três coletores por participantes durante 3 dias de coleta, sendo o quarto dia reservado para possíveis entregas em atraso. A partir das amostras recebidas, foram confeccionadas duas lâminas de cada amostra pelo método de Kato-Katz, procedimento realizado e descrito por (Katz, 2018). Este material foi repassado aos laboratoristas da Secretária Estadual de Saúde de Sergipe que, depois de secas, foram lidas e identificadas como positivas ou negativas através da microscopia comum (objetiva 10x).

Após a confecção e leitura das lâminas pela técnica de Kato-Katz, as amostras seriam lidas, posteriormente, pela método parasitológico de Ritchie, sendo assim, para garantir uma maior preservação das amostras e consequentemente dos objetos de análise (ovos, cistos e vermes adultos) foi adicionado nestas amostras formol a 10\%, garantindo assim uma melhor conservação. As amostras contendo formol foram encaminhadas para o Laboratório de Entomologia e Parasitologia Tropical, localizado na Universidade Federal de Sergipe, confeccionadas duas lâminas pelo método de Ritchie, descrito por Colombo et al., (2018) e lidas através da microscopia óptica comum (objetivas 10x e 40x). Os moradores que se apresentaram parasitados foram encaminhados ao serviço de saúde local para tratamento adequado. Após identificar a prevalência das parasitoses os resultados foram associados às condições socioambientais locais visando relacioná-las à ocorrência no povoado. Todos os moradores elou responsáveis que participaram da pesquisa foram convidados a responder um questionário, com perguntas relacionadas as condições sociais e demográficas. O questionário também foi utilizado para caracterizar a situação 
socioeconômica das famílias e as condições sanitárias das moradias (destino dos dejetos, existência de banheiros e fonte de abastecimento de água) dentre outros.

\subsection{Análise Estatística}

Para as análises estatísticas foram utilizados: as frequências absoluta e relativa, o teste de Qui-Quadrado de Igualdade e Razão de Prevalência, visto que em estudos transversais com desfechos binários, a associação entre exposição e o desfecho é estimada pela Razão de Chance (Razão de Prevalência) (Coutinho, et al., 2008). O nível de significância foi estabelecido em $5 \%$ ( $\mathrm{p}<0,05)$ e o intervalo de confiança foi de 95\%. O banco de dados foi construído no programa Microsoft Excel (Windows 2013). Todas as análises estatísticas foram realizadas no programa BioEstat (Versão 5.0).

O Teste Qui-Quadrado de Igualdade é um dos testes não-paramétricos mais utilizados em pesquisas biológicas, destinando-se a comprovar se as variáveis independentes de uma amostra interferem nos resultados da variável dependente. (Leal, 2018).

\subsection{Aspectos Éticos}

O estudo foi aprovado pelo Comitê de Ética e Pesquisa com o seguinte parecer ( $\left.\mathrm{n}^{\mathrm{o}} 3.214 .538\right)$ de 21 de março de 2018. Todos os participantes foram esclarecidos quanto ao objetivo de estudo, a participação voluntária, a garantia ao anonimato e todos assinaram o Termo de Consentimento Livre Esclarecido (TCLE).

\section{Resultados}

Foram cadastrados 187 indivíduos residentes do povoado, sendo 160 examinados. Ao todo foram distribuídos 439 coletores durante os 3 dias de inquérito. Destes, 436 recolhidos e permitiram a realização dos exames parasitológicos $\left(1^{\circ}\right.$ dia: 159 amostras; $2^{\circ}$ dia: 147 amostras; $3^{\circ}$ dia: 130 amostras). A tabela 1 indica, respectivamente, a prevalência das parasitoses encontradas no povoado através das técnicas analisada.

Tabela 1. Prevalência da esquistossomose mansoni e das enteroparasitoses encontradas em residentes do Povoado Siebra, município de Maruim, Sergipe, Brasil, 2018.

\begin{tabular}{ccccccccc}
\hline & \multicolumn{4}{c}{ Kato-Katz } & & \multicolumn{4}{c}{ Ritchie } \\
\multicolumn{1}{c}{ Espécies } & Positivos & Negativos & Positivos & \multicolumn{2}{c}{ Negativos } \\
\hline S. mansoni & 79 & 49,37 & 81 & 50,62 & 69 & 43,12 & 91 & 56,87 \\
T. trichiura & 20 & 12,50 & 140 & 87,50 & 58 & 36,25 & 102 & 63,75 \\
E. vermicularis & 1 & 0,62 & 159 & 99,37 & 13 & 8,12 & 147 & 91,87 \\
Ancilostomídeos & 7 & 4,37 & 153 & 95,62 & 2 & 1,25 & 158 & 98,75 \\
Hymenolepis nana & 4 & 2,50 & 156 & 97,50 & - & - & - & - \\
E. histolytica & - & - & - & - & 87 & 54,37 & 73 & 45,62 \\
G. lamblia & - & - & - & - & 126 & 78,75 & 34 & 21,25 \\
E. coli & - & - & - & - & 109 & 68,12 & 51 & 31,87 \\
B. hominis & - & - & - & - & 122 & 76,25 & 38 & 23,75 \\
E. nana & - & - & - & - & 154 & 97,25 & 6 & 3,75 \\
\hline
\end{tabular}

Fonte: Autores. 
Em relação à variável sexo 74 pertenciam ao sexo masculino e 86 ao sexo feminino. Para a análise da prevalência por faixa etária foram obtidas um total de 53 amostras referentes à faixa etária $0 \mid-15$ anos, 98 amostras da faixa etária de $16 \mid-$ 60 anos e 9 amostras referentes à faixa etária dos > 60 anos. Conforme o sexo e faixa etária, observou-se uma maior positividade para o sexo masculino e nas faixas etárias ( 0 a 15 e > 60 anos) quando analisados pelo método de Kato-Katz e o sexo feminino e a faixa etária > 60 anos quando analisados pelo método de Ritchie (Tabelas 2 e 3).

Tabela 2. Distribuição dos casos positivos, de acordo com a faixa etária, dos moradores dos Povoado Siebra, município de Maruim, Sergipe, Brasil, 2018.

\section{FAIXA ETÁRIA}

\begin{tabular}{|c|c|c|c|c|c|c|c|c|c|c|c|c|}
\hline \multirow{4}{*}{ Espécies } & \multicolumn{6}{|c|}{ Kato-Katz } & \multicolumn{6}{|c|}{ Ritchie } \\
\hline & \multicolumn{6}{|c|}{ Positivos } & \multicolumn{6}{|c|}{ Positivos } \\
\hline & \multicolumn{2}{|c|}{0 a 15 anos } & \multicolumn{2}{|c|}{16 a 60 anos } & \multicolumn{2}{|c|}{$>60$ anos } & \multicolumn{2}{|c|}{0 a 15 anos } & \multicolumn{2}{|c|}{16 a 60 anos } & \multicolumn{2}{|c|}{$>60$ anos } \\
\hline & $\mathbf{N}$ & $\%$ & $\mathbf{N}$ & $\%$ & $\mathbf{N}$ & $\%$ & $\mathbf{N}$ & $\%$ & $\mathbf{N}$ & $\%$ & $\mathbf{N}$ & $\%$ \\
\hline S. mansoni & 28 & 52,83 & 44 & 44,89 & 7 & 77,77 & 25 & 47,16 & 36 & 36,73 & 8 & 88,88 \\
\hline T. trichiura & 8 & 15,10 & 11 & 11,22 & 1 & 11,11 & 24 & 45,28 & 30 & 30,61 & 4 & 44,44 \\
\hline E. vermicularis & 1 & 1,88 & 0 & 0 & 0 & 0 & 3 & 5,66 & 7 & 7,14 & 3 & 33,33 \\
\hline Ancilostomídeos & 3 & 5,66 & 3 & 3,06 & 1 & 11,11 & 0 & 0 & 1 & 9,82 & 1 & 11,11 \\
\hline H. nana & 1 & 1,88 & 3 & 3,06 & 0 & 0 & - & - & - & - & - & - \\
\hline E. histolytica & - & - & - & - & . & - & 32 & 60,37 & 48 & 48,97 & 7 & 77,77 \\
\hline G. lamblia & - & - & - & - & 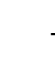 & - & 40 & 75,47 & 77 & 78,57 & 9 & 100,0 \\
\hline E. coli & - & - & - & - & . & - & 49 & 92,45 & 56 & 57,14 & 4 & 44,44 \\
\hline B. hominis & - & - & - & - & . & - & 41 & 77,35 & 73 & 74,48 & 4 & 88,88 \\
\hline E. nana & - & - & - & - & 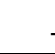 & - & 51 & 96,22 & 94 & 95,91 & 9 & 100,0 \\
\hline
\end{tabular}

Fonte: Autores. 
Tabela 3. Distribuição dos casos positivos, de acordo com o sexo, dos moradores dos Povoado Siebra, município de Maruim, Sergipe, Brasil, 2018.

\begin{tabular}{|c|c|c|c|c|c|c|c|c|}
\hline & \multicolumn{8}{|c|}{ SEXO } \\
\hline & \multicolumn{4}{|c|}{ Kato-Katz } & \multicolumn{4}{|c|}{ Ritchie } \\
\hline \multirow[t]{3}{*}{ Espécies } & \multicolumn{4}{|c|}{ Positivos } & \multicolumn{4}{|c|}{ Positivos } \\
\hline & \multicolumn{2}{|c|}{ Masculino } & \multicolumn{2}{|c|}{ Feminino } & \multicolumn{2}{|c|}{ Masculino } & \multicolumn{2}{|c|}{ Feminino } \\
\hline & $\mathbf{N}$ & $\%$ & $\mathbf{N}$ & $\%$ & $\mathbf{N}$ & $\%$ & $\mathbf{N}$ & $\%$ \\
\hline S. mansoni & 39 & 52,70 & 40 & 46,51 & 36 & 49,31 & 33 & 37,93 \\
\hline T. trichiura & 12 & 16,21 & & 9,30 & 21 & 28,76 & 37 & 42,52 \\
\hline E. vermicularis & 0 & 0 & 1 & 1,16 & 5 & 6,84 & 8 & 9,19 \\
\hline Ancilostomídeos & 6 & 8,10 & 1 & 1,16 & 1 & 1,36 & 1 & 1,14 \\
\hline Hymenolepis nana & 1 & 1,35 & 3 & 3,48 & - & - & - & - \\
\hline E. histolytica & - & - & - & - & 28 & 38,35 & 59 & 67,81 \\
\hline G. lamblia & - & - & - & - & 52 & 71,23 & 74 & 85,05 \\
\hline E. coli & - & - & - & - & 32 & 43,83 & 77 & 88,50 \\
\hline B. hominis & - & - & - & - & 47 & 64,38 & 75 & 86,20 \\
\hline E. nana & - & - & - & - & 86 & 94,53 & 68 & 78,16 \\
\hline
\end{tabular}

Fonte: Autores.

A utilização do teste Qui-Quadrado de associação nomeou algumas das variáveis independentes que mostraram associação significativa com a positividade para a infecção humana por Schistosoma mansoni. Estas variáveis foram: Sexo, naturalidade, zona e ajuda financeira governamental. A variável número de contatos foi agrupada em duas categorias: sim ou não. Abaixo estão representados os resultados da análise (tendo como base as variáveis independentes acima mencionadas) a partir de 160 moradores que aderiram a pesquisa e a relação entre essas variáveis com a prevalência da infecção pelo Schistosoma mansoni. De acordo com os resultados, a faixa etária de 20 aos 29 anos (58,62\%) e o sexo masculino (52,70\%) se mostraram mais acometidos pelo Schistosoma mansoni para essas variáveis o teste de qui quadrado se mostrou significativo ( $\mathrm{p}=0,0271$ e $\mathrm{p}=0,0500)$. Os moradores naturalizados em Maruim (59,52\%), residentes da zona rural $(54,09 \%)$ e que recebem ajuda financeira governamental $(80,53 \%)$ se mostraram mais cometidos pelo Schistosoma mansoni apresentando um valor altamente significativo $(\mathrm{p}=0,001)$ (Tabela 4). 
Tabela 4. Distribuição da população em relação à positividade por Schistosoma mansoni com o método KK, no município de Maruim/Sergipe, 2018.

\begin{tabular}{|c|c|c|c|c|c|}
\hline VARIÁVEIS & Positivo & & Negativ & & $\mathbf{P}$ \\
\hline Sexo & $\mathbf{N}$ & $\%$ & $\mathbf{N}$ & $\%$ & \\
\hline Masculino & 39 & 52,70 & 35 & 47,29 & $\mathrm{p}=0,0500$ \\
\hline Feminino & 40 & 46,51 & 46 & 53,48 & \\
\hline \multicolumn{6}{|l|}{ Idade } \\
\hline Até 9 anos & 16 & 35,55 & 29 & 64,55 & \multirow{5}{*}{$\mathrm{p}=0,0271$} \\
\hline 10 a 19 anos & 15 & 46,87 & 17 & 53,12 & \\
\hline 20 a 29 anos & 17 & 58,62 & 12 & 41,37 & \\
\hline 30 a a 39 anos & 14 & 45,16 & 17 & 54,83 & \\
\hline$>40$ anos & 17 & 48,57 & 18 & 51,42 & \\
\hline \multicolumn{6}{|l|}{ Naturalidade } \\
\hline Maruim & 50 & 59,52 & 34 & 40,47 & \\
\hline Alagoas & 29 & 38,15 & 47 & 61,84 & $\mathrm{p}=0,0001$ \\
\hline \multicolumn{6}{|l|}{ Zona } \\
\hline Rural & 46 & 62,16 & 28 & 37,83 & \multirow[t]{2}{*}{$\mathrm{p}=0,0001$} \\
\hline Urbana & 33 & 38,37 & 53 & 61,86 & \\
\hline \multicolumn{6}{|l|}{$\begin{array}{c}\text { Recebe ajuda do } \\
\text { Governo? }\end{array}$} \\
\hline Sim & 45 & 80,35 & 11 & 19,64 & \multirow[t]{2}{*}{$\mathrm{p}=0,0001$} \\
\hline Não & 34 & 32,69 & 70 & 67,30 & \\
\hline
\end{tabular}

$\mathrm{x}^{2}$ de igualdade: $\mathrm{p}<0,05$. Fonte: Autores.

A tabela abaixo apresenta os resultados da análise e a relação entre as variáveis condições sanitoambientais, origem da água consumida, dados educacionais com a prevalência da infecção pelo Schistosoma mansoni. Mostraram significativos os resultados obtidos de acordo com as variáveis tipo de banheiro $(\mathrm{p} \leq 0,0001)$ e as variáveis educacionais, ambas com o $(\mathrm{p} \leq 0,0001)$ (Tabela 5). 
Tabela 5. Distribuição da população em relação à positividade por Schistosoma mansoni com o método KK, no município de Maruim/Sergipe, 2018.

\begin{tabular}{|c|c|c|c|c|c|}
\hline \multirow[t]{2}{*}{ VARIÁVEIS } & \multirow{2}{*}{$\begin{array}{c}\text { Positivo } \\
\mathbf{N}\end{array}$} & \multicolumn{3}{|c|}{ Negativo } & \multirow[t]{2}{*}{$P$ valor } \\
\hline & & $\%$ & $\mathbf{N}$ & $\%$ & \\
\hline \multicolumn{6}{|l|}{ Destino do Esgoto } \\
\hline Ligada a Rede & 5 & 71,42 & 2 & 28,57 & $\mathrm{p}=0,0157$ \\
\hline Céu Aberto & 44 & 46,80 & 50 & 53,19 & \\
\hline Fossa & 30 & 50,84 & 29 & 49,16 & \\
\hline \multicolumn{6}{|l|}{ Destino do Lixo } \\
\hline Coleta de lixo & 50 & 45,45 & 60 & 54,54 & $\mathrm{p}=0,0009$ \\
\hline Céu Aberto & 29 & 58,00 & 21 & 42,00 & \\
\hline \multicolumn{6}{|l|}{ Tipo de Banheiro } \\
\hline Interno com água & 65 & 46,42 & 75 & 53,57 & \\
\hline Externo com água & 11 & 73,33 & 4 & 26,67 & $\mathrm{p} \leq 0,0001$ \\
\hline Não Possui & 3 & 60,00 & 2 & 40,00 & \\
\hline \multicolumn{6}{|l|}{ Origem da água } \\
\hline Encanada & 14 & 82,35 & 3 & 17,64 & \\
\hline Poço & 7 & 87,50 & 1 & 12,50 & $\mathrm{p}=0,0007$ \\
\hline Cisterna & 58 & 42,96 & 77 & 57,03 & \\
\hline \multicolumn{6}{|l|}{ Conhece sobre doença? } \\
\hline Sim & 32 & 76,19 & 10 & 23,80 & $\mathrm{p} \leq 0,0001$ \\
\hline Não & 47 & 39,83 & 71 & 60,16 & \\
\hline $\begin{array}{l}\text { Sabe como pega } \\
\text { doença? }\end{array}$ & & & & & \\
\hline Sim & 18 & 81,81 & 4 & 18,18 & $\mathrm{p} \leq 0,0001$ \\
\hline Não & 55 & 41,98 & 76 & 58,01 & \\
\hline
\end{tabular}

$\mathrm{x}^{2}$ de igualdade: $\mathrm{p}<0,05$. Fonte: Autores.

A Tabela 6 constata as variáveis epidemiológicas analisadas por meio do teste Razão de Chance, através do método parasitológico de Kato-Katz. Os indivíduos sob o maior risco para adoecer são indivíduos do sexo masculino (56,7\%), em idade (9 a >40 anos), que residem em área rural (54,1\%), recebem ajuda financeira governamental (75,6\%), com constante contato com águas naturais $(52,8 \%)$, que recebem informações sobre as parasitoses $(76,1 \%)$ e que haviam sido tratados $(70,9 \%)$. 
Tabela 6. Distribuição da positividade para Schistosoma mansoni de acordo com os fatores de risco associados. Povoado Siebra para o método de Kato-Katz, Maruim/SE, 2018.

\begin{tabular}{|c|c|c|c|c|c|c|c|}
\hline \multirow[t]{2}{*}{ Variáveis } & & \multicolumn{2}{|c|}{ Positivos } & \multicolumn{2}{|c|}{ Negativos } & \multirow[b]{2}{*}{ Odds Ratio } & \multirow[b]{2}{*}{ CI $95 \%$} \\
\hline & & Freq. & $\%$ & Freq. & $\%$ & & \\
\hline \multirow[t]{2}{*}{ Sexo } & Masculino & 42 & 56,7 & 32 & 43,2 & 1,7382 & 0,9281 a 3,2554 \\
\hline & Feminino & 37 & 43,0 & 49 & 56,9 & & \\
\hline \multirow[t]{2}{*}{ Zona } & Rural & 33 & 54,1 & 28 & 45,9 & 1,35 & 0,716 a 2,575 \\
\hline & Urbana & 46 & 46,4 & 53 & 53,6 & & \\
\hline
\end{tabular}

Recebe Ajuda do

Governo

\begin{tabular}{lllllll} 
Sim & 34 & 75,6 & 11 & 24,4 & 4,808 & 2,12 a 10,449 \\
\hline Não & 45 & 39,1 & 70 & 60,9 & \\
\hline
\end{tabular}

\section{Recebeu \\ Informação}

Sim

$32 \quad 76,1$

10

23,9

4,834

2,172 a 10,759

\begin{tabular}{lllllll}
$\begin{array}{l}\text { Sobre } \\
\text { Esquistossomose }\end{array}$ & $\mathbf{a}$ & Não & 47 & 39,8 & 71 & 60,2 \\
\hline
\end{tabular}

\section{Sabe como pega a \\ doença}

\begin{tabular}{cccccccc} 
& Sim & 18 & 75,5 & 4 & 25,0 & 6,218 & 1,993 a 19,397 \\
\cline { 2 - 8 } Teve a doença & Não & 55 & 41,9 & 76 & 58,1 & & \\
\cline { 2 - 8 } & Sim & 9 & 56,2 & 7 & 43,8 & 0,607 & 0,193 a 1,906 \\
\hline & Não & 36 & 67,9 & 17 & 32,1 & & \\
\hline
\end{tabular}

\begin{tabular}{llllllll}
$\begin{array}{l}\text { Apresentou } \\
\text { sintomas }\end{array}$ & Sim & 46 & 61,3 & 29 & 38,6 & 1,106 & 0,545 a 2,241 \\
\cline { 2 - 8 } & Não & 33 & 58,9 & 23 & 41,1 & & \\
\hline \multirow{2}{*}{ Foi tratado } & Sim & 22 & 70,9 & 9 & 29,1 & 2,316 & 0,98 a 5,474 \\
\cline { 2 - 8 } & Não & 57 & 51,3 & 54 & 48,7 & & \\
\hline
\end{tabular}

Fonte: Autores.

\section{Discusão}

No presente estudo, foi realizado exames parasitológicos de fezes nos moradores do povoado Siebra, localizado na zona rural do munícipio de Maruim, além de ressaltar dados sociodemográficos, econômicos, sanitoambientais e educacionais. O estudo procurou reunir e entender toda a dinâmica de transmissão na comunidade, e a partir daí, subsidiar meios de intervir nessa comunidade bem como, gerar dados para o planejamento de ações governamentais. Foram realizados 436 exames por ambas as técnicas (Kato-Katz e Ritchie). Vários autores (Faust,1936; Pereira, 2017; Kato \& Miura,1968; Katz, et al., 1968; Vale, et al., 2013) expõem que o sucesso do diagnóstico das parasitoses depende da qualidade da coleta das fezes, da técnica utilizada para análise e, principalmente, do número de amostras analisadas.

A localidade em estudo participa do Programa de Controle da Esquistossomose mansoni da vigilância epidemiológica que tem como principais objetivos: prevenir a ocorrência de formas graves da doença; reduzir a proporção de exames positivos e evitar a dispersão da endemia (Brasil, 2014). Os resultados revelam que o povoado se encontra distante de alcançar as metas 
determinadas pelo Ministério da Saúde, visto que o tratamento se baseia através da administração medicamentosa nos indivíduos acometidos pelas parasitoses e não levam em conta o conhecimento dos fatores de risco associados à distribuição o que caracteriza um grave problema de saúde pública.

Nossos resultados vão de encontro aos achados por Forero \& Carreno, (2017) de acordo com o exposto, mostrou que a técnica de Ritchie possui adequada sensibilidade a presença de ovos e cistos nas fezes, sendo essa circunstância é ponderável se levarmos em conta que, no Brasil, a esquistossomose mansônica e as enteroparasitoses constituem verminoses comuns. Tal fato ficou bem evidenciado através da metodologia que escolhemos e pela apreciação estatística. Nossa investigação, de caráter eminentemente prático, conduz à dedução de que o método de Ritchie tem méritos em relação aos diagnósticos razoavelmente seguros ou inquéritos epidemiológicos confiáveis, estando em foco as parasitoses analisadas.

Os resultados expostos nesse trabalho vão de encontro com os descritos por Barbosa, et al., (2012) na região Nordeste que expõem, que 45,5\% dos municípios de Pernambuco apresentaram positividade considerada moderada para esquistossomose, e em concordância com o trabalho realizado em Aracaju, Sergipe descrito por Barros, et al., (2017) onde pôde-se obter uma positividade para Schistosoma mansoni de 37,74\%.

A existência do rio denominado Ganhamoroba, que corta todo o município de Maruim e precisamente o povoado estudado junto ao frequente hábito de nadar, tomar banho, atravessar rios/córregos e pescar, estes, sendo os principais motivos do contato com águas naturais citados pelos moradores podem estar, diretamente, associados as altas taxas de infeção e serem considerados fatores que contribuem para a manutenção e permanência da doença no município. Em estudos realizados por Coura-Filho et al., (1994) e por Santos, (2013) o lazer (nadar/pescar) foi o principal motivo de contato dos residentes das áreas por eles estudadas, respectivamente.

O resultado deste estudo mostra uma similaridade numa investigação realizada por Nunes, et al., (2006), sobre o perfil epidemiológico da esquistossomose no Rio de Janeiro e por Santos, et al., (2016) no município de Simão Dias, estado de Sergipe, ambos constataram que a prevalência da infecção foi maior em indivíduos do sexo masculino. Segundo Gomes, et al., (2016) o maior acometimento do sexo masculino pode, também, estar relacionada a baixa procura as ações preventivas, visto que, a participação em uma intervenção de saúde pode ser interpretada como uma demonstração de fraqueza, sensibilizando o mito ancestral de que o homem, "mais forte e/ou resistente, não adoece".

O sexo feminino e a faixa etária > 60 anos estão entre os indivíduos mais afetados pelas enteroparasitoses quando analisados pelo método de Ritchie. Estes resultados fortalecem os expostos por Oliveira, (2015), pois mostra uma maior positividade no sexo feminino para as enteroparasitoses enfatizando que a alta positividade nesse sexo provém da prática de atividades domésticas em áreas potencialmente infectada e pelo ato de ingestão durante a preparação dos alimentos, mais precisamente alimentos consumidos de maneira crua. Tal acontecimento é similar aos resultados expostos no estudo realizado por Quintans \& Santos, (2008) no bairro Santa Maria, no estado de Sergipe onde a faixa etária $>40$ anos se mostrou mais prevalente tanto para a esquistossomose quanto para as enteroparasitoses, seguido das crianças e adolescentes. Corroborando o achado do presente estudo Santos, et al., (2017) num estudo realizado em Aiquara, no estado da Bahia, avaliou idosos da faixa etária > 60 anos e encontrou altas positividade dessas parasitoses uma média de 30,5\%, sendo a faixa de $>80$ anos a de maior positividade $38,1 \%$.

Os autores enfatizam a importância desses estudos, demonstrando que as enteroparasitoses ainda são relevantes na questão de saúde pública, bem como nas faixas etárias mais avançadas. Melo, et al., (2019) afirma que por questões financeiras ou culturais muitos idosos ainda praticam atividades de pesca podendo ser utilizada como um fator para a elevação da positividade da infecção, pois nesta, os pescadores são mais expostos porque desenvolvem atividades com partes de seu corpo dentro d'água. 
A região Nordeste reflete a pior situação de precariedade do Brasil. Uma em cada quatro pessoas nas áreas rurais vive na pobreza (Ifad, 2016). Outro aspecto a evidenciar refere-se à situação financeira dos participantes da pesquisa, cuja a maioria dos indivíduos acometidos recebem ajuda financeira governamental. É importante notar que a maioria das pessoas infectadas pela esquistossomose mansoni vive em regiões marginalizadas, de baixa renda e com recursos limitados, com saneamento inadequado. Nossos achados estão em conformidade com outras investigações. De acordo com Cantanhede, (2010) no estado do Maranhão, relacionado ao status econômico do indivíduo e a prevalência dessas parasitoses, este, reforça a existência de fortes relações entre o risco e a intensidade de infecção por esquistossomose em relação ao status socioeconômico dos indivíduos. Moreira, et al., (2011) afirma que a renda familiar baixa dificulta às famílias o acesso a serviços de saúde de qualidade e a determinados tratamentos e a medicamentos, ficando os mesmos vulneráveis aos serviços oferecidos pelo sistema de saúde pública, muitas das vezes, precários.

Os indivíduos que receberam informações sobre as parasitoses exibiram altas positividades. Esses resultados corroboram com os encontrados por (Guimarães \& Tavares-Neto, 2006; Santos \& Ribeiro, 2010). Para Melo et al., (2011) uma orientação à população que leve em conta o grau de instrução é indispensável para prevenir a doença. Segundo Santos \& Ribeiro, (2010) para combater a esquistossomose as medidas preventivas e o controle biológico são as melhores opções a curto prazo, porém ressaltamos que a importância da educação em saúde deveria ser abordada com maior frequência.

Observa-se que as atividades de nadar, tomar banho, pescar e atravessar rios e córregos apresentaram os maiores percentuais de positividade. Essa situação foi constatada por (Santos, 2013; Barreto, et al., 2015; Melo, et al., 2019) ao mencionar que atividades na água como pesca, banho, atravessar e nadar foram estatisticamente associados à infecção por $S$. mansoni. As condições sociais, ambientais e sanitárias nas quais vivem os moradores presumem uma associação da ocorrência dessas parasitoses. A falta de esgotamento, abastecimento de água do sistema público, destino dos dejetos e a precária cobertura da coleta seletiva de lixo tem como efeito a contaminação dos rios, tornando-se prognósticos associados às infecções parasitárias. Evidencia-se ainda, que a precária cobertura de água potável, no povoado, é precária, tornando-se comum o armazenamento de águas em cisternas e poços para consumo e/ou uso doméstico. Gizaw, et al., (2019) coloca que é necessário que a comunidade tenha acesso a instalações de gestão de resíduos e de saneamento contribuindo com a redução da contaminação das águas dos rios.

Em relação ao conhecimento relacionado as parasitoses para Melo, et al., (2011) uma orientação à população que leve em conta o grau de instrução é indispensável para prevenir a doença. Segundo Santos, et al., (2010) para combater a esquistossomose as medidas preventivas e o controle biológico são as melhores opções a curto prazo, porém ressaltamos que a importância da educação em saúde deveria ser abordada com maior frequência.

\section{Conclusão}

O presente estudo revela que, a localidade estudada apresenta condições favoráveis para a transmissão da esquistossomose mansoni e das enteroparasitoses. A inexistência e ineficácia do saneamento básico, coleta seletiva de lixo, baixo conhecimento e renda dos moradores dificulta o acesso aos serviços básicos de saúde bem como os recorrentes hábitos comportamentais inadequados, podem ser considerados fatores que colaboram com a existência e permanência destas infecções, além de contribuir para o aumento da prevalência, transformando-se em um local de transmissão para esquistossomose mansoni e para as enteroparasitoses.

Torna-se urgente e inadiável que medidas de saúde sejam tomadas em conjunto com ações sociais, sanitárias, ambientais e educacionais com o objetivo de acabar elou reduzir essas doenças. A implementação de programas voltado para a educação sanitária e em saúde associada com o reforço da vigilância podem contribuir com o panorama da saúde, para isso são 
necessários esforços envolvendo as secretarias de saúde e de educação, além de envolver toda a comunidade como corresponsáveis pela saúde do município.

A abordagem metodológica utilizada neste estudo identificou os riscos e as populações prioritárias para as ações de intervenção possibilitando um processo de reorganização das práticas/estratégias do programa de controle das referidas parasitoses. Todavia, de nada adianta a intervenção com programas de controle se a população não se conscientizar com a importância da prevenção em saúde, no que tange a higienização das mãos, dos alimentos e o não uso de coleções hídricas potencialmente contaminadas, uma vez que as doenças parasitárias podem acarretar consequências graves.

Financiamento: O apoio ao projeto foi fornecido pela Coordenação de Aperfeiçoamento de Pessoal de Nível Superior.

Interesses concorrentes: Nenhum declarado

Aprovação ética: Os protocolos de estudo foram revisados e aprovados pelo Comitê de Pesquisa Ética da Universidade Federal de Sergipe, Brasil (protocolos n $\left.{ }^{\circ} 3.214 .538\right)$.

\section{Referências}

Barbosa, CS; Gomes, ECS; Santos, PHS. (2012) Manual prático para o diagnóstico e controle da Esquistossomose. Recife, Editora Universitária da UFPE; ;6(4):53-8. retrieved from http://saude.sp.gov.br/resources/sucen/homepage/downloads/arquivos-esquistossomose/manualesquistossomose.pdf

Barreto, AVMS.; Santiago, RT.; Silva, MBA (2015) Analysis of Schistosomiasis mansoni positivity in endemic Health Regions in the State of Pernambuco, Brazil, 2005-2010. Epidemiol. Serv. Saúde, Brasília, v. 5. N.5. p-1-9. doi: https://doi.org/10.5123/S1679-49742015000100010.

Barros, RC.; Gomes, KVG; Casotti, CA. (2017). Prevalence of intestinal parasitosis and associated factors among the elderly. Rev. Bras. Geriatr. Gerontol. Rio de Janeiro,20(2). doi: doi.org/10.1590/1981-22562017020.160137.

Brasil. (2017). Ministério da Saúde. Secretaria de Vigilância em Saúde. Guia prático para operacionalização da Campanha Nacional de Hanseníase, $\begin{array}{lllll}\text { Verminoses, Tracoma e } & \text { Esquistossomose. } & \text { Brasília, } & \text { [Internet] } & \text { 22(12):2575-2583. retrieved }\end{array}$ http://portalarquivos2.saude.gov.br/images/pdf/2017/novembro/22/Guia-Pratico-de-Hanseniase-WEB.pdf

Brasil. (2014). Ministério da Saúde. (2014) Vigilância da esquistossomose mansoni: Diretrizes técnicas. Secretaria de Vigilância em Saúde. 4 ed. (4):53-8. retrieved from http://portalarquivos2.saude.gov.br/images/pdf/2014/dezembro/01/Vigilancia-esquistossomose-mansoni-2014.pdf

Brasil. Ministério da Saúde. Vigilância e controle de moluscos de importância epidemiológica: Programa de Vigilância e Controle da Esquistossomose (PCE), 2. ed. - [Internet]. Brasília: Editora do Ministério da Saúde. 178 p.: il. Color. retrieved from http://bvsms.saude.gov.br/bvs/publicacoes/vigilancia_controle_moluscos_import_epidemio_2ed.pdf

Brasil. (2012). Plano integrado de ações estratégicas de eliminação da esquistossomose como problema de saúde pública, tracoma como causa de cegueira e controle das geohelmintoses. retrieved from http://bvsms.saude.gov.br/bvs/publicacoes/plano_integrado_acoes_estrategicas_2011_2015.pdf

Cantanhede, SPD. (2010). Esquistossomose Mansônica no Maranhão: relações com variáveis socioeconômicas e ambientais. 101f. 2010. (Dissertação de Mestrado) - Escola Nacional de Saúde Pública Sergio Arouca, Rio de Janeiro. v49. N.4. p-1-12. retrieved from https://www.arca.fiocruz.br/handle/icict/34390.

Colombo, MS; Moura, RGF; Ferreira, G L S; et al., (2018). Enteroparasitos em amostras de Lactuca sativa em um município no estado de Minas Gerais. Arq. Ciênc. Vet. Zool. UNIPAR, 19(1):194-204. doi: 10.25110/arqvet.v21i1.2018.6624.

Coura-Filho, P.(1994). The Use of Risk Factor Determination for Schistosomiasis inEndemic Areas in Brazil. Cad. Saúde Públ., Rio de Janeiro, 10 (4): 464472, Oct/Dez, 51: 70. P-1-12. doi: doi.org/10.1590/S0102-311X1994000400006

Coutinho, LMS; Scazufca, M.; Menezes, PR. (2008). Métodos para estimar razão de prevalência em estudos de corte transversal. Rev Saúde Pública. 42(6):992-87. retrieved from https://www.scielosp.org/article/rsp/2008.v42n6/992-998/

Dunn, JC.; Turner, HC.; Tun, A.; Anderson, RM. (2016) Epidemiological surveys of, and research on, soil-transmitted helminths in Southeast Asia: a systematic review. Parasites \& vectors. 91:547-8.doi: doi.org/10.1186/s13071-016-1310-2.

Faust, EC.(1939). Comparative efficiency of various technics for the diagnosis of protozoa and helminths in feces. J. Parasit. 25(1): 7-16. retrieved from https://www.cabdirect.org/cabdirect/abstract/19402900137

Fernandez, MA. (2009). A dispersão e a distribuição atual dos moluscos hospedeiros naturais da esquistossomose no Brasil. Cad. Saúde Públ. Jun/Oct, 58:547. https://doi.org/10.1590/S0102-311X2002000500039

Ferreira, I. F.(2017). O exame parasitológico das fezes. Estudo comparativo das principais técnicas. Rev.Soc. Bras. Med. Trop.547-8. retrieved from https://www.scielo.br/pdf/rsbmt/v17n4/08.pdf 
Fochesatt-Filho, L; Barros, E. (2013). Medicina Interna na Prática Clínica. Porto Alegre: Artmed .24(3):507-16. retrieved from https://scienti.minciencias.gov.co/gruplac/jsp/visualiza/visualizagr.jsp?nro=00000000000464

Forero, JCG; Carreno, AMG. (2017). Comparación de sensibilidad y especificidad de dos técnicas de diagnóstico directo: Kato-Katz-saf y Ritchie-frick (formol-gasolina) en examen coproparasitológico para la identificación de estadios infectivos de geohelmintos en población infantil en edad preescolar y escolar. Rer. Med. 25(2). DOI: 10.18359 / rmed.3088

Gizaw, Z; Adane, T; Azanaw, J; Ayenew, A; et al., (2018). Childhood intestinal parasitic infection and sanitation predictors in rural Dembiya, northwest Ethiopia. Environmental Health and Preventive Medicine..v42. n.7.7. doi: 10.1186/s12199-018-0714-3.

Gomes, AC L; Galindo, JM.; Lima, NN; et al.,. (2016). Prevalência e carga parasitária da esquistossomose mansônica antes e depois do tratamento coletivo em Jaboatão dos Guararapes, Pernambuco. Epidemiol. Serv. Saude, Brasília, 25(1): 7-16. http://dx.doi.org/10.5123/S1679-49742016000200003

Guimarães, ICS, Tavares-Neto, J. (2006). Transmissão urbana da esquistossomose em crianças de um bairro de Salvador, Bahia. Rev Soc Bras Med Trop. setout.;39(5):451-5. retrieved from https://repositorio.ufba.br/ri/bitstream/ri/2650/1/v7n3a12.pdf

IBGE, Instituto Brasileiro de Geografia e Estatística, 2019. Cities @ Sergipe [Internet]. retrieved from https://www.ibge.gov.br/

IFAD. (2016). Fundo Internacional de Desenvolvimento Agrícola. Investindo nas populações rurais do Brasil. https://www.fida.org.br/

Kato, K.; Miura, M. (1954) Comparative examinations. Jap. J. Parasit. 9(1):194-204. retrieved from https://dps.mn.gov/divisions/bca/bca-divisions/forensicscience/Pages/comparative-examinations.aspx

Katz, N. (2018) Inquérito Nacional de Prevalência da Esquistossomose mansoni e Geohelmintoses/Naftale Katz. 19(12):1504-14. retrieved from https://www.arca.fiocruz.br/bitstream/icict/25662/2/Inqu\%C3\%A9rito\%20Nacional\%20de\%20Preval\%C3\%AAncia\%20da\%20Esquistossomose\%20mansoni $\% 20 \mathrm{e} \% 20 \mathrm{Geo}-$ helmintoses.pdf

Katz. N; Chaia, G. (1968). Coprological diagnosis of Schistosomiasis. I. Evaluation of quantitative technique. Rev. Inst. Med. Trop. S. Paulo, . v. 5. N.5. -1-9. retrieved from https://www.cabdirect.org/cabdirect/abstract/19692900833

Leal, IB. (2018). Fatores de risco e análise espacial da infecção por Schistosoma mansoni em escolares de área endêmica no estado de Sergipe, Brasil. 2018. 103f. Dissertação (Mestrado em Biologia Parasitária) - Universidade Federal de Sergipe, São Cristóvão- SE, 26 (11): 23-30. https://ri.ufs.br/bitstream/riufs/11555/2/IANE_BRITO_LEAL.pdf

Machado, W. (2017). Prevalência da esquistossomose mansoni e geohelmintoses em escolares do município de Malhador, Sergipe. Dissertação (Mestrado em Biologia Parasitária). Atlas. retrieved from http://lareferencia.info/vufind/Record/BR_8a2816b2932544ec5d0135265a971055

Melo, AGS; Irmão JJ M.; Jeraldo, VLS.; Melo, CM. (2019). Esquistossomose mansônica em trabalhadores da pesca de área endêmica de Alagoas. Revista Anna Nery. 22(12):2575-2583. https://doi.org/10.1590/2177-9465-ean-2018-0150

Melo, AGS; Melo, CM.; Oliveira, CCC; Oliveira, D. S; et al., (2011). Esquistossomose em Área De Transição Rural-Urbana: Reflexões Epidemiológicas. Cienc Cuid Saude.doi: :10.4025/cienccuidsaude.v10i3.12479

Moreira, ML; Valadão, AF; Martins, J. (2011). Prevalence of mansonic schistosomiasis and associated factors to its occurrence in students from the rural area of Inhapim-MG, 2008. Rev. Bras. Farm.(4):53-8. doi: 10.1186/s13071-021-04692-8.

Nunes FC; Costa MCE; Filhote M.I,F; Sharapinn, M. (2006). Perfil epidemiológico da esquistossomose mansoni no bairro Alto da Boa Vista, Rio de Janeiro. Rev. Saúde Coletiva.. 209-228. retrieved from http://www.cadernos.iesc.ufrj.br/cadernos/images/csc/2005_3/artigos/CSC_2005-3_fabiola.pdf

Oliveira, JW. (2015). Análise e comparação da sensibilidade e especificidade entre diferentes métodos de diagnóstico para Schistosoma mansoni: gradiente salino, helmintex®, centrífugo-sedimentação, Kato-Katz e teste rápido urina (POC-CCA). (Dissertação de Mestrado) - Atlas. retrieved from https://repositorio.ufmg.br/handle/1843/BUBD-9WHFJU

Pereira, GLT; Ribeiro, CA; Costa, IO; Silva, JNC; et al., (2017). Prevalência de Infecções Parasitárias Intestinais Oriundas de Crianças Residentes em Áreas Periféricas, Município de Juazeiro do Norte - Ceará. Rev. Interfaces, v42. n.7 -7. DOI:10.16891/2317-434X.v5.e14.a2017.pp21-27

Pereira, G.LT; Ribeiro, C A; Costa, IO; Silva, JNC.; et al., (2015). Frequência de parasitoses intestinais: um estudo com crianças de uma creche de Santa Cruz do Sul - RS. Cinergis, 25(1): 7-16. DOI: 10.17058/cinergis.v16i2.6426.

Quintans, J; Santos, R. (2008). Avaliação do Programa de Controle de Esquistossomose no Bairro Santa Maria, Aracaju, Sergipe, sob a Perspectiva Farmacêutica. Revista da Fapese, v.4, n.2, p. 63-82, jul./dez. https://doi.org/10.1590/S0037-86822011000100020

Reuter, CP. (2015). Frequência de parasitoses intestinais: um estudo com crianças de uma creche de Santa Cruz do Sul - RS. Cinergis, (7): e023420. DOI: http://dx.doi.org/10.17058/cinergis.v16i2.6426

Sánchez, A; Munoz, M; Gómez, N. (2017) Molecular Epidemiology of Giardia, Blastocystis and Cryptosporidium among Indigenous Children from the Colombian Amazon Basin. Front. Microbiol., 28(1). https://doi.org/10.3389/fmicb.2017.00248

Santos, AD; Lima, ACR.; Santos, MB; Alves, JAB; Góes, M. (2016). Análise espacial para identificação de áreas de risco para esquistossomose mansoni no Estado de Sergipe, Brasil, 2005-2014. Rev. da Sociedade Brasileira de Medicina Tropical, n52;v.20. DOI:10.1590/S0102-311X2011000500008

Santos, AD. (2013). Fatores de risco associados à distribuição da infecção por Schistosoma mansoni Na Comunidade Do Bairro Santa Maria, Aracaju-SE. . Dissertação (Mestrado em Biologia Parasitária). Atlas. retrieved from https://www.arca.fiocruz.br/bitstream/icict/4041/2/000007.pdf 
Research, Society and Development, v. 10, n. 5, e26310514538, 2021

(CC BY 4.0) | ISSN 2525-3409 | DOI: http://dx.doi.org/10.33448/rsd-v10i5.14538

Santos, AD.; Santos, MB.; Santos, PGR.; Barreto, AS.; et al., (2016). Análise espacial e características epidemiológicas dos casos de esquistossomose mansônica no município de Simão Dias, Nordeste do Brasil. Rev. Patol. Trop. 19(12):1504-14. DOI:10.5216/rpt.v45i1.39978

Santos, CS.; Ribeiro, AS. (2010). Study of the biological control of schistosomiasis in public schools Sergipe state. REMPEC - Ensino, Saúde e Ambiente, 26 (11): 23-30. file:///C:/Users/alex_/Documents/CEA/21127-Texto\%20do\%20Artigo-77539-1-10-20180920.pdf

Siqueira, TS; Celestino, AO; Silva, JRS. (2020). Factors associated with cure and abandonment of leprosy treatment: A reflective analysis. Journal Research, Society e Development. 22(12):2575-2583. DOI: 10.33448 / rsd-v10i1.11615

Vale, EP.; Sampaio, TIS.; Távoras, JA. (2013). Análise comparativa de três métodos parasitológicos de fezes em uma escola do município de Macapá-AP. Revista de Biologia e Ciências da Terra. 209-228. https://docplayer.com.br/25845213-Analise-comparativa-de-tres-metodos-parasitologicos-de-fezes-emuma-escola-do-municipio-de-macapa-ap.html 